\title{
Evaluating C-RAN Fronthaul Functional Splits in Terms of Network Level Energy and Cost Savings
}

\author{
Checko, Aleksandra; Popovska Avramova, Andrijana; Berger, Michael Stübert; Christiansen, Henrik \\ Lehrmann
}

\author{
Published in: \\ Journal of Communications and Networks
}

Link to article, DOI:

10.1109/JCN.2016.000025

Publication date:

2016

Document Version

Peer reviewed version

Link back to DTU Orbit

Citation (APA):

Checko, A., Popovska Avramova, A., Berger, M. S., \& Christiansen, H. L. (2016). Evaluating C-RAN Fronthaul Functional Splits in Terms of Network Level Energy and Cost Savings. Journal of Communications and Networks, 18(2), 162-172. https://doi.org/10.1109/JCN.2016.000025

\section{General rights}

Copyright and moral rights for the publications made accessible in the public portal are retained by the authors and/or other copyright owners and it is a condition of accessing publications that users recognise and abide by the legal requirements associated with these rights.

- Users may download and print one copy of any publication from the public portal for the purpose of private study or research.

- You may not further distribute the material or use it for any profit-making activity or commercial gain

- You may freely distribute the URL identifying the publication in the public portal 


\title{
Evaluating C-RAN fronthaul functional splits in terms of network level energy and cost savings
}

\author{
Aleksandra Checko*, Andrijana P. Avramova*, Michael S. Berger and Henrik L. Christiansen
}

\begin{abstract}
The placement of the complete baseband processing in a centralized pool results in high data rate requirement and inflexibility of the fronthaul network, which challenges the energy and cost effectiveness of the Cloud Radio Access Network (C-RAN). Recently, redesign of the C-RAN through functional split in the baseband processing chain has been proposed to overcome these challenges. This paper evaluates, by mathematical and simulation methods, different splits with respect to network level energy and cost efficiency having in the mind the expected quality of service.

The proposed mathematical model quantifies the multiplexing gains and the trade-offs between centralization and decentralization concerning the cost of the pool, fronthaul network capacity and resource utilization. The event-based simulation captures the influence of the traffic load dynamics and traffic type variation on designing an efficient fronthaul network.

Based on the obtained results, we derive a principle for fronthaul dimensioning based on the traffic profile. This principle allows for efficient radio access network with respect to multiplexing gains while achieving the expected users' quality of service.
\end{abstract}

Index Terms: C-RAN; functional split; multiplexing gain; energy efficiency.

\section{INTRODUCTION}

Striving towards cost and energy efficient next generation of mobile communications, C-RAN designates a leading technology for the Radio Access Network (RAN) architecture [1]. C-RAN inherits the design of a distributed Base Station (BS), where the Remote Radio Head (RRH) ${ }^{1}$ is separated and connected via fiber to the baseband processing server, called Baseband Unit (BBU). The baseband processing is gathered in a centralized pool, which facilitates advanced multi-cell cooperation techniques. Thus the radio resource management is more efficient as the network is flexible and scalable to the temporally and spatial fluctuations of the mobile traffic. The centralization is further enhanced with cloud computing [2], providing elasticity, virtualization with possibility for multitenancy among operators. The computational resources can be pooled and dynamically allocated to a virtual BS, which brings cost-effective hardware and software design [3].

\footnotetext{
* The first two authors contributed equally to this work.

This work was partially sponsored by the $7^{\text {th }}$ Framework Programme for Research of the European Commission HARP project, under grant number HARP318489.

A. Checko, A. P. Avramova, M. S. Berger and H. L. Christiansen are with the DTU Fotonik, Department of Photonics Engineering, Technical University of Denmark, Kgs. Lyngby, Denmark, email: [amch, apop, msbe, hlch]@fotonik.dtu.dk.

A. Checko is also with MTI Radiocomp, Hillerød, Denmark, email: aleksandra.checko@mtigroup.com.

${ }^{1} \mathrm{~A}$ RRH is consisted of radio frequency equipment and antenna
}

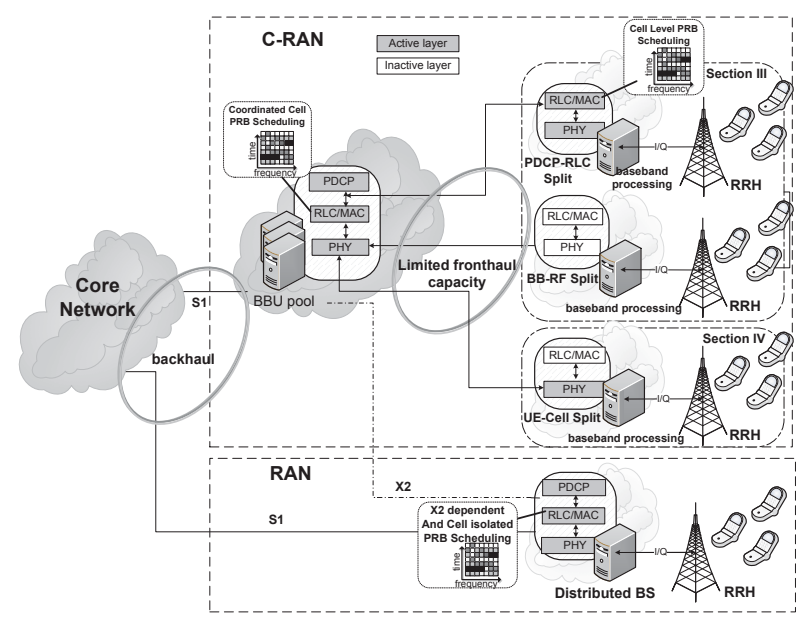

Fig. 1. RAN Architecture for different functional splits.

With the current interface definition, the fronthaul network (connecting the BBU pool and the RRHs) relies on technologies that are capable of supporting high capacity, such as optical fiber. For example, in case of Common Public Radio Interface (CPRI) fronthaul interface, for a $20 \mathrm{MHz}$ bandwidth and $2 \times 2$ MIMO antenna system, the data rate required by the links for downlink in one sector is 2457.6 Mbps, where only $150 \mathrm{Mbps}$ is offered to a single cell users on the air interface [4]. The transport efficiency on the fronthaul links is highly reduced as the interface is independent of the traffic load while dependent of the number of antennas used. As such, massive Multiple Input Multiple Output (MIMO) will further challenge the cost-effectiveness of the C-RAN architecture.

The high cost due to the stringent requirement of such fronthaul infrastructure has lead to investigation of alternative points of separating the functionality in the baseband processing chain ([4], [5], [6]) as illustrated in Fig. 1. The future definitions of the fronthaul interface need to realize the benefits of centralization and cloud computation at highest possible level, while relaxing the bandwidth and latency requirements.

In this paper we discuss and analyze different split points in terms of multiplexing gains, which relates to cost and energy efficiency of the transport network. First we present a quantitative study of different splits based on teletraffic theory. Afterwards we provide simulation based analysis where more detailed traffic models have been applied in order to derive in-depth and detailed study of a particular functional split. The research elaborated in this paper suggests that hybrid deployments should be considered in order to optimally balance the multiplexing gains, spectral efficiency and cost of the fronthaul infrastructure. This paper gives indication of the multiplexing gains through network 
dimensioning, while ensuring satisfying quality of service to the end user. The results obtained substantially influence the operator's decision of the radio access network deployments.

This structure of the paper is illustrated in Fig. 1. Section II defines the multiplexing gains considered in this paper and elaborates on possible split points in the baseband processing chain. Section III evaluates the functional splits using direct routing model based on multi-dimensional loss system, while Section IV reports the results from the discrete event-based simulation analysis. Finally, concluding remarks are presented in Section V.

\section{METHODS FOR QUANTIFYING MULTIPLEXING GAINS}

In this section we explain the sources and definition of multiplexing gains in mobile networks. We discuss possible functional splits and multiplexing gains they enable. Moreover, we explain the methodologies taken in this article to quantify multiplexing gains. Last, we provide an overview of the state-of-theart on quantifying multiplexing gains.

\section{A. Background and terminology}

The art of network/BBU dimensioning is to provide a network that is cost-effective for the operator and at the same time provides a reasonable Quality of Service (QoS) for users. By allowing many users and base stations to share network resources, multiplexing gain can be achieved, as they will request peak data rates at different times. The multiplexing gain comes from traffic independence and from 1) burstiness of the traffic, and 2) the tidal effect - daily traffic variations between office and residential cells [7]. Given the fact that cells from metropolitan area can be connected to one BBU pool (maximum distance between RRH and BBU is required to be within $20 \mathrm{~km}$ ) it is realistic to account for office and residential cells to be included. The tidal effect is one of the main motivations for introducing C-RAN [7].

As for any other shared resources, multiplexing enables to serve the same amount of users with less equipment. Multiplexing gain indicates the savings that comes from the less equipment required to serve the same number of users. In this way the cost of deploying BBU pools and fronthaul links (CAPital EXpenditure (CAPEX)) will be lower. That will lead to energy savings, as fewer BBU units and fronthaul links need to be supplied with electricity (OPerating EXpenditure (OPEX)).

\section{B. Multiplexing gains for different functional splits}

In a traditional base station or in a base station with RRH, for each cell, the baseband processing resources are statically assigned to the RRH, as shown in Fig. 2a. In C-RAN, shown in Fig. $2 d$ the baseband units are shared in a virtualized BBU pool, hence it is expected that in C-RAN the amount of processors needed to perform baseband processing will be lower comparing to the RAN. The CPRI protocol is constant bit rate, independent of user activity. Hence, there is no multiplexing gain on fronthaul links. We call this split BB-RF as is separates baseband and radio frequency functionalities.

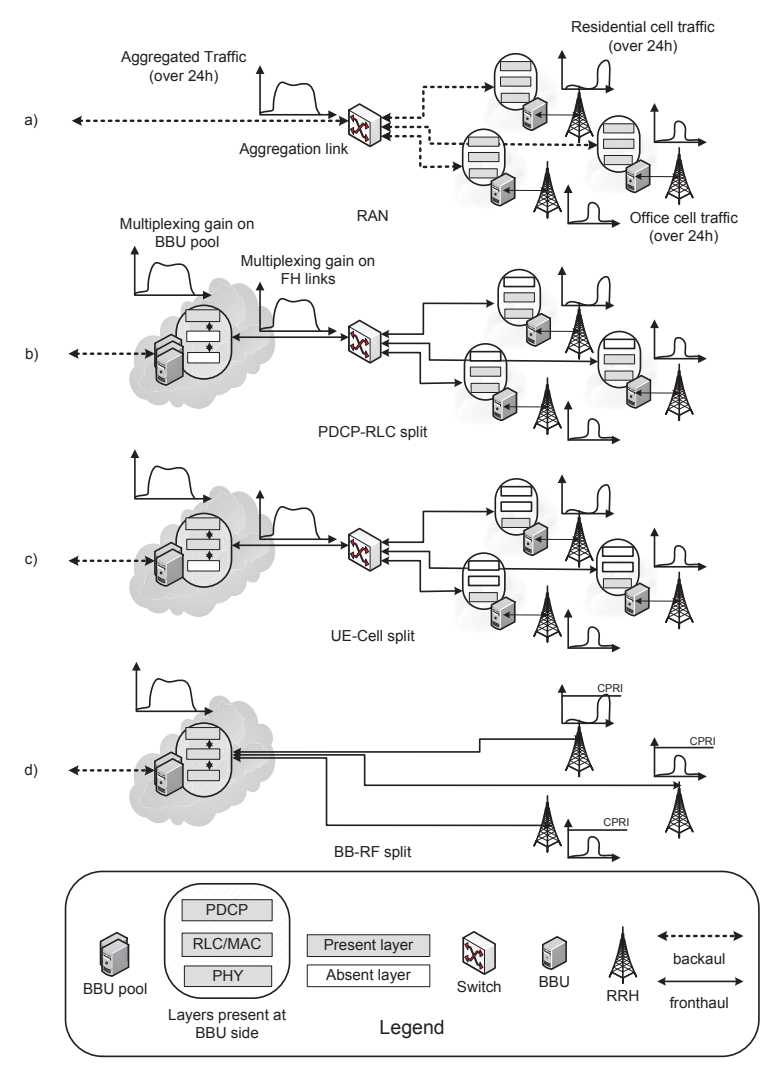

Fig. 2. Possible multiplexing gains depending on base station architecture.

Before year 2013, to address the challenge of optimizing the fronthaul bit rate and flexibility various compression techniques were considered. As a more disruptive methods were needed to achieve higher data rate reduction, nowadays (from year 2013) a new split between RRH and BBU functionality is under an extensive analysis [4], [8], [9]. In the analysis reported by this paper we focus on three functional splits as indicated in Fig. 2: BB-RF, discussed above, UE-Cell and PDCP-RLC. With the $U E$-Cell split (separating user and cell specific functionalities), shown in Fig. 2c traffic between RRH and BBU will be traffic dependent, hence we can expect multiplexing gain both on BBU resources but also on fronthaul links. However, fronthaul latency requirements are tight - $250 \mu s$ [4]. For PDCP-RLC split, shown in Fig. 2b, the majority of data processing will be executed at the cell sites, only a small portion of it will be done in a pool, hence a marginal BBU pool multiplexing gain. However, a variable bit rate traffic will be transmitted on the fronthaul links, hence a possibility for a multiplexing gain on the fronthaul. Moreover, this split allows for higher fronthaul latency - $30 \mathrm{~ms}$ [4]. This split leaves the MAC scheduling and PHY functionality to reside at the RRH, which limits the possibility for joint PHY processing and joint scheduling for multicell cooperation.

For the heterogeneous cases, when some of the cells are served by RAN, some by C-RAN with different splits, different multiplexing gain will be achieved for fronthaul and different for the BBU. Therefore the total cost of the network will vary. We evaluate this trade off in Section III. Generally, the more 
Table 1. Multiplexing gains (MG) looking at traffic-dependent resources.

\begin{tabular}{l|l|l} 
RAN Architecture & BBU & links \\
\hline RAN & 1 (no MG) & $\begin{array}{l}\text { As in UE-Cell } \\
\text { split for links }\end{array}$ \\
\hline PDCP-RLC split & Section III & Section III \\
\hline UE-Cell split & Section IV & Section IV \\
\hline BB-RF & Section III, IV & 1 (no MG)
\end{tabular}

processing is centralized, the higher savings on BBU cost, but higher burden on fronthaul links. On the other hand, the more functionalities are left at the cell side, the lower savings on BBU but at the same time the lower cost of fronthaul.

We differentiate the multiplexing gain on BBU pool and links with the new functional splits. Table 1 shows dependencies between the values.

\section{Methodology}

A base station performs functions that are both traffic independent (e.g. Fast Fourier Transform (FFT)) as well as traffic dependent (processing user and control data, e.g. modulation). In this work we look at traffic dependent resources (throughput-dependent). To measure the multiplexing gain we use a method, inspired by [10] and [8]. In all the equations we refer to the multiplexing gains as $M G$. To evaluate the multiplexing gain on BBUs we compare the baseband processing resources - BBResources $R A N$ - needed in a RAN (base station architecture with or without RRH) to the pooled baseband processing resources in a C-RAN - BBResources $B$ BUpool, as shown in equation (1). In this way, the multiplexing gain helps to define rules of thumb for BBU dimensioning and estimate cost savings.

$$
M G_{B B U}=\frac{\sum_{\text {cells }} \text { BBResources } R A N}{\text { BBResources }}
$$

To evaluate the multiplexing gain on the fronthaul links we compare the total link resources on a single link to the aggregated link resources, as shown in the equation (2). In this way, the multiplexing gain helps to define rules of thumb for fronthaul dimensioning and to evaluate the cost savings of the network.

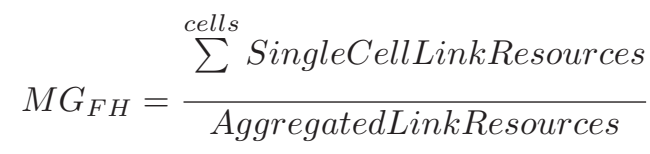

Link resources specify sufficient bandwidth for a given deployment. They can be defined in several ways, as providing the peak throughput requested by users is costly: 1) 95th percentile of requested throughput (used in Section IV-B), 2) 95th percentile confidence intervals of mean of resources (used in Section III), 3) peak requested throughput averaged over given time (used in Section IV-A) and 4) link data rate for which the application layer delay is acceptable (used in Section IV-B).

Teletraffic theory has been used for planning and administration of real telecommunications systems even when the first telephone system became commercially available. The theory provides mathematical models of telecommunications system as to perform analysis of the interrelationships among resource provisioning, random traffic demand, and quality of service [11]. The basic concept in the models is the stochastic nature of the traffic such that the performance of the system is described through probabilities: traffic, call or time congestion, the average sojourn time of the services, etc. An overview of different mathematical models, their characteristics and relevance is provided in [12], while a broad application of different models for modeling and dimensioning of mobile networks is given in [13]. Teletraffic theories have been used to calculate an overbooking factor [10] that dimensions the link, based on the definition of an effective bandwidth [13]. They provide an important indication when the fundamentals of the networks are studied. Additionally, teletraffic systems based on multi-dimensional Markov model, can capture the session level dynamics in the network and the relation of baseband processing and radio resource requirements. With this, we are able to derive a mathematical model that can be used to initially investigate flexible and heterogeneous design on the fronthaul network, and evaluate the costs due to processing requirements, fronthaul capacity and overall efficiency. In order to show how much the capacity requirements on the fronthaul network can be relaxed, we have evaluated the trade-offs between the two most opposite cases: PDCP-RLC, where the traffic on the fronthaul follows the dynamics on the traffic demand on the air interface, and the BB-RF, where the traffic on the fronthaul is constant. Additionally, we evaluate the importance of centralizing resource scheduling for spectral efficiency on air interface.

However, teletraffic theories focus on well-defined traffic models, such as ON - OFF source traffic e.g. Interrupted Poisson Process, Interrupted Bernoulli Process. As such, they do not capture all the aspects of real-life networks. In current and future mobile network there is a big variety of applications and the traffic varies throughout the day depending on cell location (office or residential). In order to capture this heterogeneity we have done part of the analysis in a discrete event-based simulator - OPNET. Such scenario with detailed and heterogeneous traffic definition is especially important to evaluate UE-Cell split. Simulations allow to capture protocol interactions and thereby observe implications of different network architectures on endto-end delay seen at the application layer. On the other hand mathematical approach allow to create simpler models that can run on lower simulation time, thereby enabling to test more extended scenarios, e.g. with more cells.

We believe that both approaches are important to compare and validate the results. In this paper we use both simulation and teletraffic approach to evaluate multiplexing gains in RAN. Results for $M G_{B B U-B B-R F}$ will be used to validate the results between two approaches, as this value will be calculated both using teletraffic approach and simulations.

\section{Related work}

Quantifying multiplexing gains has been addressed by the research and industry community. In [14] Werthmann et al. prove that significant multiplexing gains can be achieved if multiple sectors are aggregated into one single cloud base station. In [15] Bhaumik et al. show that the centralized architecture can potentially result in savings of compute resources by exploiting the 
variations in the processing load across base stations. In [16] Namba et al. analyze statistical multiplexing gain as a function of cell layout. In [17] Madhavan et al. quantify the multiplexing gain of consolidating WiMAX base stations in different traffic conditions. The gain increases linearly with network size and it is higher when base stations are experiencing higher traffic intensity. On the contrary, based on teletraffic theory, in [18] Liu et al. analyzed that lighter load can increase the statistical multiplexing gain in virtual base station pool. Moreover, multiplexing gain reaches significant level even for the medium-size pools and the increase in gain for larger pools in negligible. In our previous work, using the definition from equation (1) we compared BBU resources in RAN to C-RAN. The network consisted of office and residential base stations. We concluded that the value of the multiplexing gain is 1.2-1.6 and depends on the percentage of office base stations in the BBU pool, reaching the peak for $30 \%$ of office and thereby $70 \%$ of residential base stations [19]. Secondly, we have compared results on multiplexing gain obtained via simulations [19] to the ones achieved with teletraffic theory [20].

However, all those works referred to the traditional - BB-RF - functional split of C-RAN. In [8] authors estimate what they define as statistical multiplexing convergence ratio on fronthaul links by averaging observed daily throughput. Calculated ratio equals to three. However, the analysis took only average network load into account and therefore can be interpreted mostly as an average daily fronthaul utilization. In this work we look at different functional splits and different, precisely defined application mixes. Moreover, on top of studying traffic to calculate multiplexing gain, we also measure delays for different BBU/fronthaul dimensioning cases.

\section{TELETRAFFIC APPROACH}

In this section we provide a quantitative analysis for the different functional splits discussed in the previous sections. In particular we consider a RAN with hybrid functional split in the fronthaul interface, namely BB-RF and PDCP-RLC split. We consider these two opposite splits, but the model can be extended for comparison of all three discussed splits. The split can be defined per cell, such that a portion of RRHs are connected via CPRI to the BBU pool while the rest is connected via the PDCP-RLC split. In order to simplify the analytical model and introduce symmetry in the RRH definition, we consider dynamic split per traffic flow, and define a portion of traffic per RRH that is carried with a specific split. As we need to define the amount of pooled resources required to carry the requested volume of traffic, a network with hybrid functional splits can be represented by an equivalent direct routing network model. Such model can be used to define numerical examples that study the statistical multiplexing advantages of pooling resources in C-RAN. The following subsections first explain how we model C-RAN using notation of teletraffic theory and then we describe the direct routing analytical model based on the multi-dimensional systems. Afterwards, we elaborate on the obtained numerical results. Further information on the model used, along with the mathematical model for the carried traffic and blocking probabilities can be found in [20].

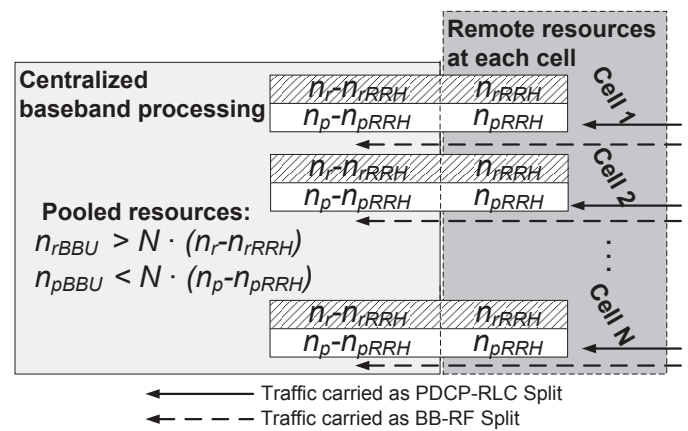

Fig. 3. Model of C-RAN with N cells, resource definition and offered traffic as PDCP-RLC and BB-RF split.

\section{A. Network layout mapping of a C-RAN deployment}

We model a C-RAN with N cells, where each cell has limited radio and baseband processing (computational) resources, denoted as $n_{r}$ and $n_{p}$. The $n_{r}$ radio resources can represent a timefrequency pair or Physical Resource Blocks (PRBs). The traffic offered with specific split at each cell is defined as a stream with mean $(A)$ and standard deviation $(s t d)$. A stream can be defined as a flow of Binomial, Poisson, or Pascal (BPP) traffic, which are generally used to describe smooth, random and peaked traffic respectively. Each stream requests a defined number of radio resources, $d^{r}$ (time-frequency pair or Physical Resource Blocks $(\mathrm{PRBs}))$ and baseband processing resources, $d^{p}$ (computational resources) for the entire connection. The pooled (radio and processing) resources are defined by $n_{r B B U}$ and $n_{p B B U}$, while $n_{r R R H}$ and $n_{p R R H}$ define the resources that are not centralized and reserved. When the overall traffic is carried as PDCP-RLC split, $n_{p B B U}=0$, and $n_{p B B U}=0$ while $n_{r R R H}=n_{r}$, and $n_{p R R H}=n_{p}$. Alternatively, when the overall traffic is carried as BB-RF split, all baseband processing is pooled, hence $n_{p B B U}<\sum_{i=1}^{N} n_{p}$, where the inequality defines the pooling gain. As the radio resource allocation is centralized, $n_{r B B U}>$ $\sum_{i=1}^{N} n_{r}$, where the inequality defines the gains from multi-cell cooperation such as Coordinated Multi-Point (CoMP). Since we are looking into hybrid deployment, the task is to define the portion of $n_{r R R H}$ and $n_{p R R H}$ as well as $n_{r B B U}$ and $n_{r B B U}$, such that the total traffic is carried with low blocking probability.

\section{B. Network with direct routing}

Our analytical model expands (enhances) the mathematical model for evaluation of multiplexing gain presented in [18] by modeling the C-RAN as a network with direct routing. Multidimensional loss system has been used in [18] order to evaluate the statistical gains from pooling virtual BS. In such a system, a single link with $n$ basic units is shared among $N$ statistically independent traffic streams. The offered traffic from the $j$-th stream is characterized by mean value $A_{j}$ (offered traffic in Erlang), and standard deviation $s t d_{j}$, while $d_{j}$ defines the required resources for the entire connection. The maximum number of processing resources that all connection from the $j$-th stream can occupy is defined by $n_{j}$, such that $\sum_{j=1}^{N} n_{j} \geq n$. Then the C-RAN system can be described by a $N$-dimensional Marko- 
vian process with state space $\underline{x}=\left(x_{1}, x_{2}, \ldots, x_{N}\right)$, where $x_{j}$ represents the number of connections of the stream from the $j$ th RRH. The following restrictions define the truncation to the state space:

$$
0 \leq x_{j} \cdot d_{j} \leq n_{j}, \quad \sum_{j=1}^{N} x_{j} \cdot d_{j} \leq n_{p}
$$

Such system is reversible and since the traffic streams are independent, the system has product form. Thus the convolution algorithm [21], which has not been considered in [18], can be applied to derive the probability of the state $p(\underline{x})$ as:

$$
p(\underline{x})=p_{1}\left(x_{1}\right) * p_{2}\left(x_{2}\right) * \ldots * p_{N}\left(x_{N}\right),
$$

By $p_{j}\left(x_{j}\right)$ we assume one dimensional Markovian process for the $j$-th stream, which is a classical loss system with full accessibility. The convolution, denoted by $*$, is done for one stream at a time, in the following manner. Let $p_{k}$ represent convolution of the first $k$ streams, then the next stream, $k+1$, is convolved as follows:

$$
\begin{aligned}
& p_{k} * p_{k+1}= \\
& \left\{p_{k}(0) \cdot p_{k+1}(0), \sum_{x=0}^{1} p_{k}(x) \cdot p_{k+1}(1-x), \ldots,\right. \\
& \left.\sum_{x=0}^{t} p_{k}(x) \cdot p_{k+1}(t-x)\right\}
\end{aligned}
$$

Here $t=\min \left(\sum_{j=1}^{k+1} n_{j}, n_{p}\right)$ and defines truncation to the state space at each step. Therefore, normalization needs to be performed at each step in order to get the true state probabilities. In order to obtain the performance metrics for the $k$-th stream, all streams except the $k$-th need to be first convolved. Let $p_{N / k}$ denotes the convolution of all streams except the $k$-th, then the carried traffic is defined as:

$$
Y_{k}=\sum_{x=0}^{n_{p}} \sum_{x_{j}=0}^{x} x_{j} \cdot p_{N / k}(x-x j) \cdot p_{k}\left(x_{j}\right)
$$

Additionally, the traffic, call and time congestion can be derived as defined in [12].

In this paper we extend the multidimensional loss system by a network with direct routing [21] in order to be able to define additional restrictions to the state space due to the considered functional splits as well as a sharing degree of PRB among RRH with respect to advanced multi-cell cooperation techniques. A network with direct routing is defined through $N$ routes $\left(R_{j}\right)$, $M$ links $\left(L_{m}\right)$ and required resources $d_{j}^{m}$ for the $j$-th stream on the $m$-th link. Each route corresponds to a traffic stream. The links have capacity $\left(l_{m}\right)$ that defines the maximum number of resources that a stream can occupy. Table 2 provides an illustration of how a network with direct routing can be defined.

The system defined by the network with direct routing still has a product form and the convolution algorithm can be applied in
Table 2. Direct routing network

\begin{tabular}{c|cccc|c}
\cline { 2 - 5 } & \multicolumn{5}{c|}{ Routes } \\
\hline Links & $R_{1}$ & $R_{2}$ & $\ldots$ & $R_{N}$ & Capacity \\
\hline$L_{1}$ & $d_{1}^{1}$ & $d_{2}^{1}$ & $\ldots$ & $d_{N}^{M}$ & $l_{1}$ \\
$L_{2}$ & $d_{1}^{2}$ & $d_{2}^{2}$ & $\ldots$ & $d_{N}^{M}$ & $l_{2}$ \\
$\ldots$ & $\ldots$ & $\ldots$ & $\ldots$ & $\ldots$ & $\ldots$ \\
$L_{M}$ & $d_{1}^{M}$ & $d_{2}^{M}$ & $\ldots$ & $d_{N}^{M}$ & $l_{M}$ \\
\hline
\end{tabular}

Table 3. Direct routing equivalent to a functional split in C-RAN

\begin{tabular}{c|cc|cc|c|cc|c}
\multicolumn{7}{c}{ Route (Stream) } \\
\cline { 2 - 9 } & \multicolumn{2}{|c|}{ Cell $_{1}$} & \multicolumn{3}{c|}{ Cell $_{2}$} & $\cdots$ & \multicolumn{3}{c}{ Cell $_{N}$} & \\
\hline Link & $R_{P}$ & $R_{C}$ & $R_{P}$ & $R_{C}$ & & $R_{P}$ & $R_{C}$ & Capacity \\
\hline$L_{A}$ & $d_{p}^{p d c p}$ & 0 & 0 & 0 & & 0 & 0 & $n_{p R R H}$ \\
\hline$L_{B}$ & $d_{r}^{p d c p}$ & 0 & 0 & 0 & & 0 & 0 & $n_{r R R H}$ \\
\hline$L_{A}$ & 0 & 0 & $d_{p}^{p d c p}$ & 0 & & 0 & 0 & $n_{p R R H}$ \\
\hline$L_{B}$ & 0 & 0 & $d_{r}^{p d c p}$ & 0 & & 0 & 0 & $n_{r R R H}$ \\
\hline$L_{A}$ & 0 & 0 & 0 & 0 & & $d_{p}^{p d c p}$ & 0 & $n_{p R R H}$ \\
\hline$L_{B}$ & 0 & 0 & 0 & 0 & & $d_{r}^{p d c p}$ & 0 & $n_{r R R H}$ \\
\hline$L_{C}$ & 0 & $d_{p}^{b b-r f}$ & 0 & $d_{p}^{b-r f}$ & & 0 & $d_{p}^{b b-r f}$ & $n_{p B B U}$ \\
\hline$L_{D}$ & 0 & $d_{r}^{b b-r f}$ & 0 & $d_{r}^{b b-r f}$ & & 0 & $d_{r}^{b b-r f}$ & $n_{r B B U}$ \\
\hline
\end{tabular}

order to derive the performance metrics for each stream. The convolution is performed by aggregating the state probabilities of one route at a time and considering the restrictions on each link due to the limited capacity:

$$
\sum_{j=1}^{N} x_{j} \cdot d_{j}^{m} \leq l_{m}, \quad m=1,2, \ldots M
$$

A direct routing equivalent to a C-RAN model with two functional splits described by Fig. 3 is given in Table 3. The routes define the stream(s) $j$ of BPP traffic associated with a RRH. The radio resources are either being scheduled at the MAC layer at the RRHs, or at the BBU pool, depending on the split. The baseband processing power required for these radio resources is reserved in the $\mathrm{RRH}$, or the BBU pool, correspondingly to the assigned radio resources. The limited amount of radio resources and processing power available need to be considered during each convolution step, and in this case they are represented by the link-restrictions. As we evaluate the performance metrics in case of different hybrid functional splits, for each stream (route) we define the resources (both radio and processing) requested at the BBU pool and the RRH. The route $R_{P}$ defines the mean offered traffic $A_{j}^{p d c p}$ and the standard deviation $s t d_{j}^{p d c p}$ of the traffic that is carried in the fronthaul network through the PDCPRLC interface at the $j$-th cell. The route $R_{C}$ defines the same characteristics $\left(A_{j}^{b b-r f}\right.$ and $\left.s t d_{j}^{b b-r f}\right)$ for the traffic that is carried through CPRI at the $j$-th cell. The capacity defines total available resources, both radio and processing, which yields the following restrictions. Link $L_{A}$ define the restrictions due to the available PRBs at each RRH $\left(n_{r R R H}\right)$, while link $L_{B}$ defines the limited processing possibilities at the RRH, defined through $n_{p R R H}$. Link $L_{C}$, and $L_{D}$ define the restrictions due to the total available radio and processing resources at the BBU pool, defined as $n_{r B B U}$ and $n_{p B B U}$ respectively. 


\section{Numerical results and discussion}

For the numerical analysis we have considered $N=100$ cells, each with total offered traffic of $A=8 \mathrm{Erl}$. and arrival rates with Poisson distribution. We have considered Poisson process with single-slot traffic $\left(d_{p}=d_{r}=1\right)$ in order to keep the studies manageable. At a fixed offered traffic, the percentage of total traffic that is carried as PDCP-RLC split is varied from 0 to $100 \%$, with a step of $10 \%$. This way the network becomes hybrid, where the deployment is partially implemented as PDCPRLC split and partially as BB-RF split. For each split percentage, we first derive the minimum amount of radio $\left(n_{r R R H}\right)$ and processing resources $\left(n_{p R R H}\right)$ required at the RRH, such that the carried traffic is equal to the offered traffic (traffic congestion below $1 \%$ ). Then we dimension the resources at the BBU pool $\left(n_{p B B U}\right)$, to ensure that the total offered traffic is carried by the network. At each split we quantify the following key performance indicators: multiplexing gains at the BBU pool, multiplexing gains on the fronthaul links and radio resource utilizations. Based on equation 1, the multiplexing gain for the computational resources at the BBU pool is defined as:

$$
M G_{B B U-\text { hybrid }}=\frac{N \cdot n_{R A N}}{N \cdot n_{p R R H}+n_{p B B U}}
$$

where $n_{R A N}$ represent the maximum processing units available at a RRH in a (traditional) RAN architecture. We only consider the multiplexing gains that come from the layers below PDCP, as all the gains of centralizing the PDCP layer are present for both functional splits. Using equation 2, the multiplexing gain at the fronthaul can be expressed through the variation of the mean value and standard deviation (defined through the state probabilities):

$$
\begin{aligned}
& M G_{F H-h y b r i d}= \\
& \frac{\sum_{j=1}^{N}\left(F \cdot\left(A_{j}^{b b-r f}+t_{n} \cdot s t d_{j}^{b b-r f}\right)+\left(A_{j}^{p d p c}+t_{n} \cdot s t d_{j}^{p d c p}\right)\right)}{\left(A_{a g g}^{p d c p}+t_{n} \cdot s t d_{a g g}^{p d c p}\right)+\sum_{j=1}^{N} F \cdot\left(A_{j}^{b b-r f}+t_{n} \cdot s t d_{j}^{b b-r f}\right)}
\end{aligned}
$$

where $t_{n}=1.96$ represent the student $\mathrm{t}$-distribution for $95 \%$ confidence interval. The notation of $A_{a g g}^{p d c p}$, st $d_{a g g}^{p d c p}$ represent the characteristics of the aggregated traffic from the PDCP-RLC split, defined as:

$$
A_{\text {agg }}^{p d c p}=\sum_{1}^{N}\left(A_{j}^{p d c p}\right), \quad s t d_{a g g}^{p d c p}=\sqrt{\sum_{j=1}^{N} s t d_{j}^{p d c p^{2}}}
$$

By $F$ we define the increase factor due to CPRI protocol nature, which has been set $F=16$, as the extreme case where 2.5 Gbps $/ 150 \mathrm{Mbps}=16$.

Implementing multi-cell cooperation, such as CoMP, leads to increased spectral efficiency, especially at the cell edges, where interference is reduced and even more used as complementary signal in case of joint transmission. In order to indicate the effects of multi-cell cooperation, we model them as a percentage of PRB sharing among cells (sharing_pct). Then we consider the PRB utilization defined through the following equation:

$$
P R B_{u t i l}=\frac{\text { carried_traffic }}{n_{r C e l l}+n_{r B B U}}
$$

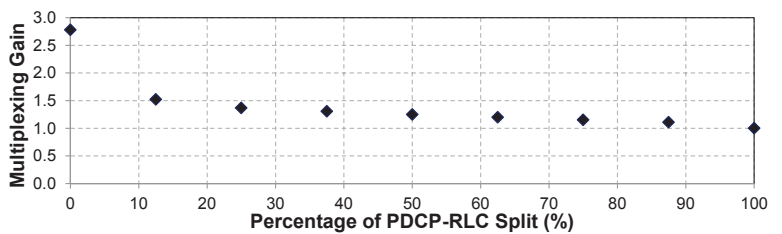

Fig. 4. Multiplexing gain in BBU pool: PDCP-RLC versus BB-RF split.

where $n_{r B B U}=\left(n_{r C e l l}-n_{r R R H}\right) \cdot$ sharing_pct, and $n_{r C e l l}$ are the PRB available at the RRH in case all traffic is carried as PDCP-RLC split. The more possibility there exist for multi-cell cooperation, the PRB are better utilized and hence less PRBs are needed in order to carry the same traffic volume. Since we consider that the total offered traffic is carried, carried_traffic $=A=8$ Erl., while sharing_pct $=30 \%$.

The results for each key indicator are given in Fig. 4 to Fig. 6. As expected, the multiplexing gains in the BBU pool is increasing as more traffic (cells with fully centralized baseband processing) is aggregated at the BBU pool. In the previous study [20] we have showed that as we aggregate more cells, the aggregated traffic has a mean value that is sum of the mean values of the aggregated traffic, but the coefficient of the variation is reduced, leading to more smooth traffic. The highest gains occur when mixing cites with more complementary traffic patters, such as office and residential areas, but significant gains are present even for random traffic as shown in Fig. 4. When the total traffic is carried through the PDCP-RLC split, there is no multiplexing gain as all resource are required at the RRHs and cannot be shared. On the other hand, in case of full centralization, there is no multiplexing gains in fronthaul links as the traffic is not depended on the load of the cells. As such the multiplexing gain for $0 \%$ of PDCP-RLC split is 1 as shown in Fig. 5. By using a factor of 16 for CPRI overload, we can see that by increasing the percent of PDCP-RLC split, gain in the fronthaul can be achieved. The maximum gain is achieved at no centralization as the traffic in the fronthaul network becomes fully load dependent. By looking equation (9), we can also conclude that the traffic load becomes high and almost constant, multiplexing gains at the fronthaul will be reduced. If the traffic load is low, and even more bursty, higher multiplexing gains can be achieved. An other important metric is the physical resource block utilization shown in Fig. 6 indicating resource efficiency gains are reduced as we reduce the centralization percentage. This is an important metric to be considered as either additional effort need to be placed in order to provide multi-cell cooperation, or the operator needs to be aware that channel utilization will be increased when reducing the percentage of centralization, in order to carry the same amount of traffic. By increasing the channel utilization, the sensitivity of the overall system to overload is reduced.

In order to evaluate the split percentage based on all three criteria described above, the following cost weight function can be defined:

$$
\text { cost }_{\text {value }}=B B U \cdot c_{b b u}+F H \cdot c_{\text {link }}+P R B \cdot c_{\text {prb }}
$$

In equation (12), $B B U$ indicates the required amount of pro- 


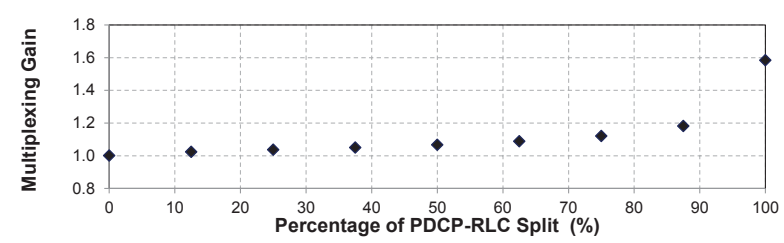

Fig. 5. Multiplexing gain at fronthaul links.

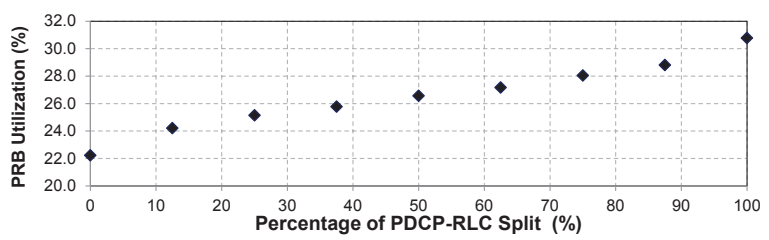

Fig. 6. PRB Utilization.

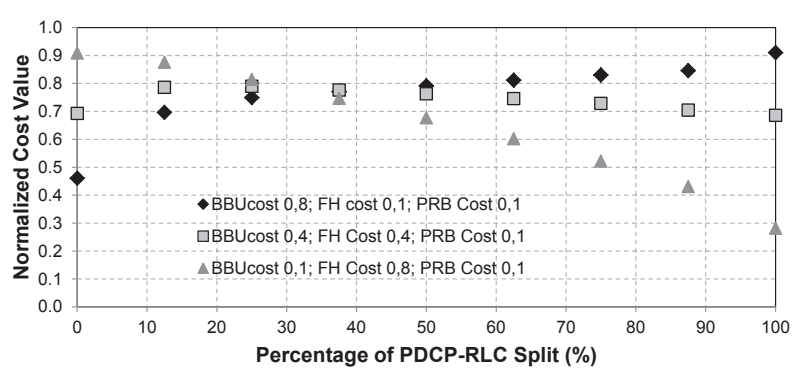

Fig. 7. Weighted cost evaluation based on gains

cessing power (computational resource required for baseband processing), $F H$ indicates the required capacity on the fronthaul links (infrastructure cost), and $P R B$ indicates the percentage of radio resource utilization (spectrum gains) and. The values of $c_{\text {link }}, c_{p r b}$ and $c_{b b u}$ represent the associated cost and are normalized such that their sum is equal to unit value. Fig. 7 illustrates how the normalized cost of the RAN depends on the weight that is placed on each of the individual gains as considered in equation 12. We do not indicate real values for the cost, as the cost of the equipment depends on the current deployment and hence influences the individual cost for the operator. This figure also shows that hybrid deployments should also be considered by the operators depending of the goal and the cost area where the operator needs to save the most. It can help to define the portion of traffic that the operator will route on a particular functional split, depending on the load of the network and the available resources.

\section{DISCRETE EVENT SIMULATION MODEL}

In the previous section we investigated multiplexing gains in PDCP-RLC and BB-RF splits using the analytical approach. In this section we study UE-Cell and BB-RF split using discrete event simulations. We want to investigate the application mix impact, therefore we vary percentage of web and video traffic, while the total offered traffic follows the daily load for each sim- ulation run.

We built an exemplary system with an architecture and protocol stack similar to the one used for our previous study [19]. Ten base stations are connected to one switch and then to a server, as presented in Fig. 8. The aggregated link represents fronthaul and BBU traffic for UE-Cell split, therefore we can calculate the multiplexing gain on fronthaul links for UE-Cell split $M G_{F H-U E-C e l l}$. $M G_{F H-U E-C e l l}$ comes straightforward from equation (2) where we quantify LinkResources as throughput or data rate. We are evaluating only traffic dependent resources, therefore the comparison between single and aggregated link resources is analogical to comparing traffic on BBUs in RAN to BBU pool in C-RAN. As a consequence, $M G_{F H-U E-C e l l}$ is the same as multiplexing gain on BBU for UE-Cell split $M G_{B B U-U E-C e l l}$ and BB-RF split $M G_{B B U-B B-R F}$. Later on in this Section we will refer to all this results as $M G_{F H-U E-C e l l}$, but the same conclusions appear to $M G_{B B U-U E-C e l l}$ and $M G_{B B U-B B-R F}$. Traffic in the BBU pool and on fronthaul links in UE-Cell split can be compared to Ethernet traffic on a MAC layer. PHY layer cell processing will be done at the cell site leaving MAC-layer-like traffic on the fronthaul. Each base station is connected with a 1 Gbps link, as this could be a radio link throughput of LTE-A and initially data rate of the aggregated link is $10 \mathrm{Gbps}$ not to create any blocking. There are three office and seven residential base stations, as this is the mix for which we observed the maximum multiplexing gain in our previous studies [19], [20]. Daily traffic load between office and residential cells varies according to [7]. We send video and web traffic according to the definitions presented in Table 4 to represent delay sensitive (ms level) and delay insensitive (s level) applications. Parameters for video traffic are based on [22] and for web traffic on [23] considering an average web page size growth between years 2007 and 2014 [24]. Values presented in the table represent traffic from 8 a.m. to 9 a.m. for office base station (lowest load observed in the system) and are multiplied for other hours and residential base stations to reflect the daily load. Simulation parameters are presented in Table 5. No QoS-aware scheduling was done, the packets were processed in First Input First Output (FIFO) manner. This simple scheduling algorithm was used to show the emphasis on traffic aggregation, not scheduling as such.

\section{A. Throughput measurements for quantifying multiplexing gains}

Given the fact that the links between the base station and the aggregating switch have a data rate of $1 \mathrm{Gbps}$, it can be seen on ns level whether a bit is sent or not. LTE scheduling is done every $1 \mathrm{~ms}$, therefore it is viable to measure not more often then once every $1 \mathrm{~ms}$. For practical reasons, in order to be able to process the results efficiently, we chose to collect data every $10 \mathrm{~ms}$. Operators will most likely not dimension their network for peak user data measured over $1 \mathrm{~ms}$, but allow some buffering, thereby saving the costs, although lowering the user data rate. Therefore we applied different averaging over simulated throughput as follows.

For each cell $c$ and for the aggregated link $a$ the data set resulting from simulations consists of $\frac{16 \text { hours }}{10 \mathrm{~ms}}=5760000$ throughput measurements $x$ measured at time $t$. We define an averaging 


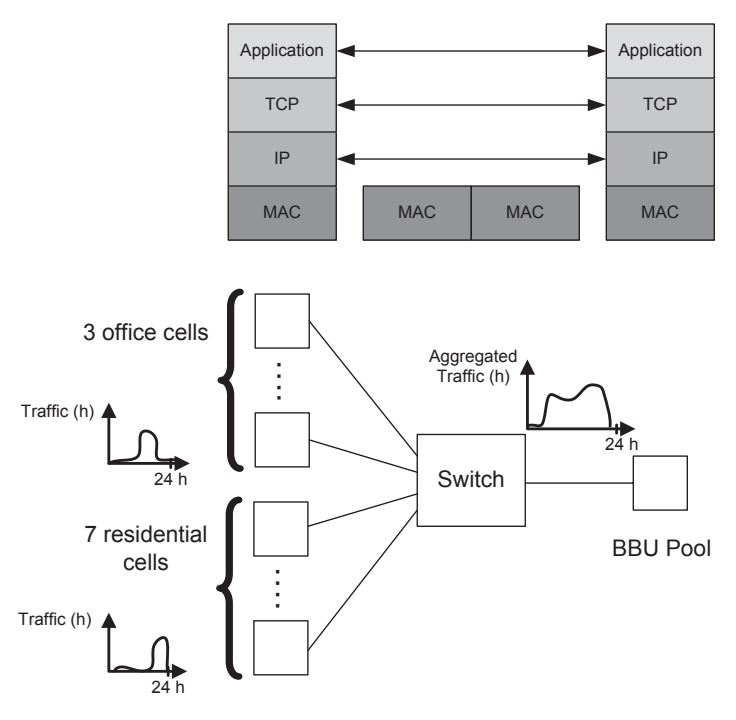

Fig. 8. Network model used for simulations.

Table 4. Traffic generation parameters for network modeling; C - Constant, E Exponential, L - log-normal, G - gamma, U - uniform

\begin{tabular}{l|l} 
Traffic Parameters & Value, Distribution \\
\hline \multicolumn{2}{c}{ Video application } \\
\hline $\begin{array}{l}\text { Frame interarrival } \\
\text { time }\end{array}$ & 10 frames/sec, C \\
\hline Frame size & $4193 \mathrm{~B}, \mathrm{C}$ \\
\hline $\begin{array}{l}\text { Duration of video con- } \\
\text { ference, 50\% cases }\end{array}$ & mean: $16 \mathrm{~s}$, variance: $5 \mathrm{~s}, \mathrm{~N}$ \\
\hline $\begin{array}{l}\text { Duration of video con- } \\
\text { ference, 25\% cases }\end{array}$ & mean: $208 \mathrm{~s}$, variance: $3364 \mathrm{~s}, \mathrm{~N}$ \\
\hline $\begin{array}{l}\text { Duration of video con- } \\
\text { ference, 25\% cases }\end{array}$ & mean: $295 \mathrm{~s}$, variance: $2500 \mathrm{~s}, \mathrm{~N}$ \\
\hline Inter-repetition time & mean: $1250 \mathrm{~s}, \mathrm{E}$ \\
\hline
\end{tabular}

Web browsing application

Page interarrival time $\quad$ mean: 28.5 s, variance: 1774966 $\mathrm{s}, \mathrm{L}$

main object size mean: $63346 \mathrm{~B}$, variance: $86205010504 \mathrm{~B}, \max 6$ $\mathrm{MB}, \mathrm{L}$

Page properties Number of embedded objects scale: 40, shape: 0.1414, $\max$ $300, \mathrm{G}$

embedded object size
mean: $142103 \mathrm{~B}$, variance: $2264446191523 \mathrm{~B}$, max $6 \mathrm{MB}, \mathrm{L}$

window (bucket) of a width $W$ such that for the samples $\left(t_{i}, x_{i}\right)$ where $i=0,1, \ldots, n$ and $t_{n}-t_{0}=W$. The averaging window size represents the networks ability to smoothen the traffic and has a similar function to a buffer. We divide 16 hours simulated time into such windows $W$ and for each of them we calculate an average throughput $y=\frac{\sum_{i=0}^{n} x_{i}}{n}$. Out of all the $y$ values, we find a maximum value of all the averages $y_{\max }$ for each cell $y_{\max , c}$ and for an aggregated link $y_{\max , a}$. Based on equation (2) we
Table 5. Simulation parameters.

\begin{tabular}{l|l} 
Parameter & Value \\
\hline $\begin{array}{l}\text { Modeler and simula- } \\
\text { tion software }\end{array}$ & OPNET 17.5.A PL3 \\
\hline Simulated time & $16 \mathrm{~h}$ \\
\hline Seeds & 24, random \\
\hline Values per statistic & $\begin{array}{l}\text { For throughput measurements ev- } \\
\text { ery } 10 \mathrm{~ms}, \text { for delay measurements } \\
\text { every } 1 \mathrm{~s}\end{array}$
\end{tabular}

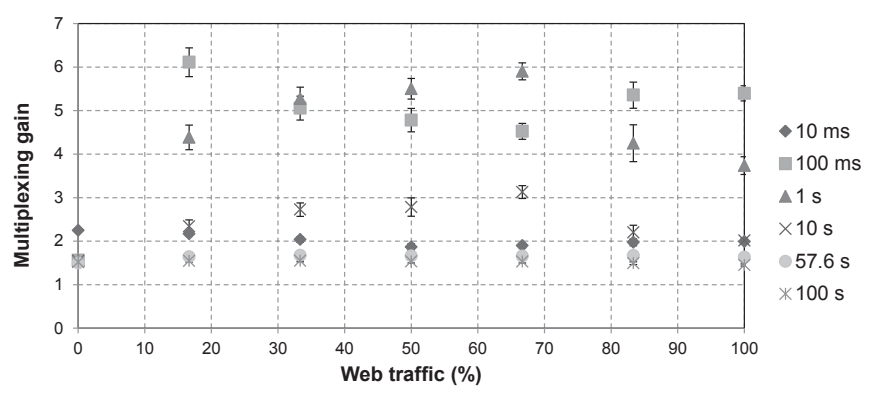

Fig. 9. Multiplexing gain for for different percentage of web traffic in the system and different throughput averaging windows: $M G_{F H-U E-C e l l}(10 \mathrm{~ms}$, no averaging) and $M G_{F H-U E-C e l l}-A V G(100 \mathrm{~ms}, 1 \mathrm{~s}, 10 \mathrm{~s}, 57 \mathrm{~s}$ and $100 \mathrm{~s})$.

calculate $M G_{F H-U E-C e l l-A V G}$ as presented in equation (13).

$$
M G_{F H-U E-C e l l-A V G}=\frac{\sum_{c=1}^{c e l l s} y_{\max , c}}{y_{\max , a}}
$$

Values of $M G_{F H-U E-C e l l-A V G}$ coming from simulations for different web and video traffic mixes are presented in Fig. 9. Confidence intervals for $95 \%$ level were calculated using the Student's t distribution. Different series present data averaged over $100 \mathrm{~ms}, 1 \mathrm{~s}, 10 \mathrm{~s}, 57 \mathrm{~s}$ and $100 \mathrm{~s}$ (averaging window $W$ ). For $10 \mathrm{~ms}$ series throughput was not averaged, only the maximum values were taken for each cell and aggregated link to compute $M G_{F H-U E-C e l l}$. Values vary for different mixes of web traffic. For no web traffic present in the network, the multiplexing gain has similar value within our averaging intervals, as video conferencing sessions have constant bit rates. As soon as web traffic is present (17-100\%) multiplexing gain varies from 1.5 to 6 depending on the averaging window.

It can be seen that multiplexing gain is very sensitive to the measurement interval. A summary of results achieved here and in our two other projects and impact on averaging is shown in a Table 6. It shows a clear dependence of the averaging period on the multiplexing gain.

In principle, if we take longer, up to infinite, averaging periods the multiplexing gain should be getting lower and reaching one, as the average bandwidth of an aggregation link will need to match the sum of average bandwidths of single links. Therefore it is not straightforward why the value is low for every $10 \mathrm{~ms}$, then increases for $100 \mathrm{~ms}$ and $1 \mathrm{~s}$ and then lowers again. This is because the Cumulative Distribution Function (CDF) of the throughput looks in a way that for $90 \%$ of the time the through- 
Table 6. Multiplexing gains for different throughput averaging windows, calculated for different projects. MG - multiplexing gain.

\begin{tabular}{|c|c|c|c|}
\hline Project & $\begin{array}{l}\text { Simulated } \\
\text { time }(\mathrm{h})\end{array}$ & $\begin{array}{l}\text { averaging } \\
\text { window } W\end{array}$ & MG \\
\hline $\begin{array}{l}\text { LTE dimension- } \\
\text { ing [25] }\end{array}$ & 1 & $1 \mathrm{~s}$ & 4 \\
\hline $\begin{array}{l}\text { C-RAN } \quad \text { MG } \\
\text { varying \% Office } \\
\text { cells[19] }\end{array}$ & 16 & $576 \mathrm{~s}$ & $1.2-1.6$ \\
\hline \multirow{6}{*}{$\begin{array}{l}\text { C-RAN MG } \\
\text { varying \% } \\
\text { Web Traffic - } \\
\text { this study }\end{array}$} & \multirow{6}{*}{16} & $10 \mathrm{~ms}$ & $1.9-2.3$ \\
\hline & & $100 \mathrm{~ms}$ & $1.6-6.1$ \\
\hline & & $1 \mathrm{~s}$ & $1.6-5.9$ \\
\hline & & $10 \mathrm{~s}$ & $1.5-3.1$ \\
\hline & & $57.6 \mathrm{~s}$ & $1.5-1.7$ \\
\hline & & $100 \mathrm{~s}$ & $1.5-1.6$ \\
\hline
\end{tabular}

put to base stations is below $100 \mathrm{Mbps}$ and aggregated throughput is below $1 \mathrm{Gbps}$, as presented on Fig. 10. This indicates, that by adequate dimensioning the multiplexing gain value can be different. Moreover, if the dimensioning was done according to the results from averaging over longer periods, the risk of dropped packets and connections would increase, as buffer sizes may be exceeded and packets may be dropped or users may not be satisfied with the delay. In this study none of the packets were dropped. The averaging was done only in post processing of the data, so actually it was not verified what would be the impact of providing only the data rates as averaged on the application level delays. For video conferencing and web browsing averaging only up to $10-100 \mathrm{~ms}$ is safe, as application layer delays should not exceed the order of magnitude of $150 \mathrm{~ms}$ and $1 \mathrm{~s}$, respectively. Delays on the application level will give an ultimate criterion for network dimensioning. We elaborate on them in the following section.

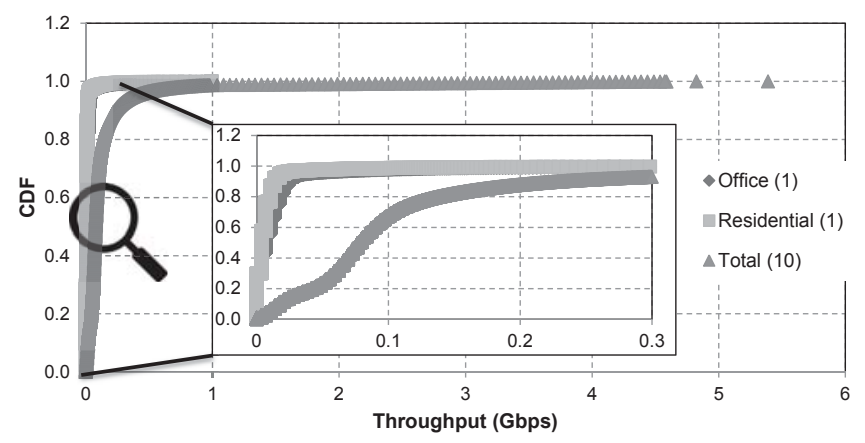

Fig. 10. CDFs of throughput for an exemplary office and residential cell as well as total throughput for all ten cells for $50 \%$ web traffic mix.

\section{B. Delay-based criteria for network dimensioning}

The ultimate criterion to dimension the discussed links and BBU pool is to assure acceptable performance on the application level. For that we check web page response time and video packet end-to-end delay via event-based simulations. For 100$400 \mathrm{Mbps}$ aggregated link data rate the delay differences are the highest and they reach the point when they become acceptable. We intentionally examined links with fine granularity of throughputs (every $50 \mathrm{Mbps}$ ) as lines and computational power can be leased with fine granularity of data rates [26]. The results are presented in Fig. 11 and 12. For web traffic 90th percentile of web page response time is below $1 \mathrm{~s}$ for link data rates $\geq 200$ Mbps. For video conferencing 90th percentile of packet end-toend delays are below $150 \mathrm{~ms}$ for link $\geq 200 \mathrm{Mbps}$.

As expected, the delays are lower when offered link data rates are higher. The impact on delay is higher for the cases with less web traffic. It is due to the fact, that the more video traffic is present in the network, the delays are more sensitive to the aggregated link bandwidth. Small change of data rate affects delay considerably, even by a factor of 10 (for $17 \%$ of web traffic - $83 \%$ of video traffic). The reason could be that video occupies a link at a constant bit rate for at least a minute, so if the links are under-dimensioned queuing occurs. We can conclude here that the more bursty the traffic is, the less sensitive is it to underdimensioning. The more video traffic present in the network the dimensioning becomes more relevant for achieving quality of service. Traffic forecast [27] predicts that in $202060 \%$ of the mobile traffic will be video, however, it will vary between light and heavy users.

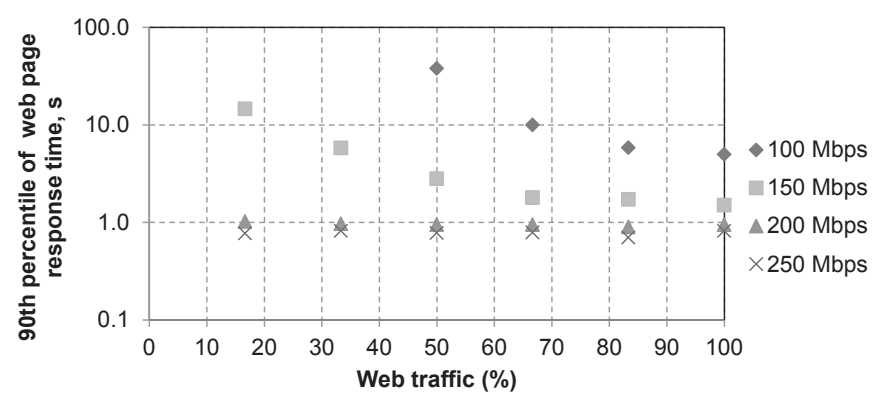

Fig. 11. 90th percentile of web page response time for different percentage of web traffic in the system and for different aggregated link data rate.

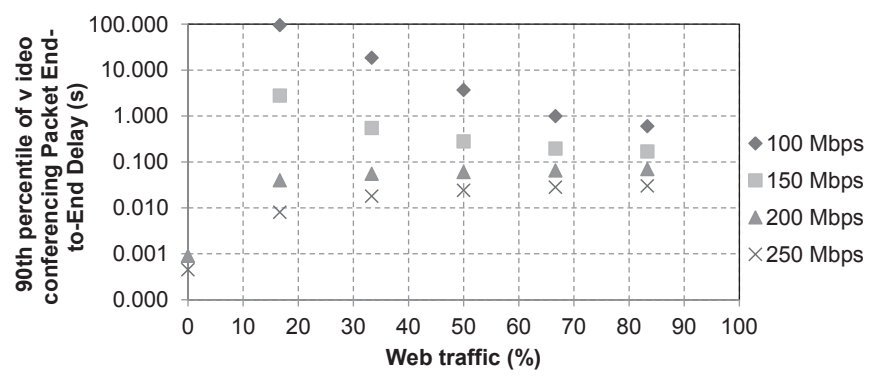

Fig. 12. 90th percentile of video conferencing packet End-to-End delay for different percentage of web traffic in the system and for different aggregated link data rate.

We analyzed the sum of the 80th, 90th and the 95th percentiles of single cells throughputs and the 80th, 90th and the 95th percentiles of an aggregated link throughput. The results are presented in Fig. 13. The more web traffic in the network 
the lower the mean traffic, but the standard deviation gets higher. Therefore the sum of 80th and 90th percentiles are getting lower. However, when we look at 80th and 90th percentiles on aggregated link, it is getting higher because the high peaks occur more often. The trend shown on Fig. 11 and 12 are the same as for sum of the 80th and the 90th percentiles on Fig. 13. The values closest to $200 \mathrm{Mbps}$, that proven to be sufficient from the delay analysis, are the 80th percentile on aggregated link and sum of 90th percentiles. The higher one needs to be supported. We can therefore conclude, that in order to dimension fronthaul links and BBU sum of 90th percentile of throughputs from fronthaul links and 80th percentile of aggregated link need to be provided (here $200 \mathrm{Mbps}$ ). In case of under-dimensioning, for higher percentages of web traffic the delay increase will be lower, as was the sum of the 80 th and the 90th percentiles.
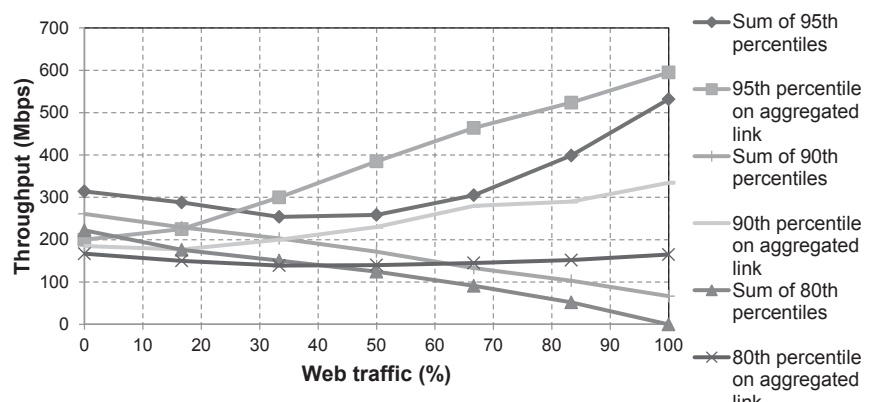

Fig. 13. 80th, 90th and 95th percentile of base stations throughput for different percentage of web traffic in the system.

These results can be used not only for quantifying multiplexing gains but also for network dimensioning providing traffic distribution with a CDF similar to the one studied here. For upgrading existing networks, operators could measure single cell throughput and calculate the 90th percentile of it and measure 80th percentile of aggregated traffic. Then depending on how many cells should belong to one BBU pool/be aggregated on a single fronthaul link, the higher number will assure the necessary capacity. If it is not met, it means that links should be upgraded to the next available data rate. For green field deployments based on traffic forecast, an operator will need to estimate what is the 90th percentile of throughput he would like to offer to the users. The challenge then would be to add those throughputs taking into account forecasted user activity. Having the sum of 90th percentile of such an aggregated traffic for each base station and 80th percentile of aggregated traffic, the capacities need to be summed considering how many cells will be aggregated on a link/BBU pool. The sum will give the desired link/BBU pool resources.

The results for the sum of 95th percentiles can be applied to the equation (2), where sufficient AggregatedLinkResources are $200 \mathrm{Mbps}$, based on delay measurements. Using this method computed $M G_{F H-U E-C e l l 95 t h}$ is in the range of 1.27 2.66 which converges with the results for $M G_{F H-U E-C e l l}$ for throughput measured every $10 \mathrm{~ms}$ (1.5-2.2). Moreover, 2.7 was the result of $M G_{B B U-B B-R F}$ concluded with the teletraffic method ( $0 \%$ of PDCP-RLC, meaning $100 \%$ of BB-RF), which confirms the thesis that $M G_{F H-U E-C e l l}=M G_{B B U-B B-R F}$ stated at the beginning of this section. This results serves as validation of the two approaches.

\section{CONCLUSIONS}

C-RAN is seen as a candidate architecture for $5 \mathrm{G}$ mobile networks because of the cost and performance benefits it offers. Due to the fact that securing fronthaul capacity to deploy fully centralized C-RAN can be costly, a careful analysis of cost and energy savings for different functional splits is of great importance. As energy and cost savings are related to the multiplexing gain, in this study we evaluate it for different functional splits.

We calculated multiplexing gains on throughput-dependent functionalities of a base station for different C-RAN functional splits: BB-RF, separating user and cell specific functions, and PDCP-RLC using two different methodologies. For given traffic definitions we gave quantitative analysis of multiplexing gains. However, the results are sensitive to the traffic profiles as well as to multiplexing gain calculation method. Therefore the main outcome of this study is to provide the trend lines that will facilitate finding an optimal trade off when fronthaul or BBU resources are more costly for an operator.

For fully centralized C-RAN - with BB-RF split - maximum multiplexing gain on BBU resources can be achieved. However, the required fronthaul capacity is the highest. Therefore this split is vital for operators with cheap access to fronthaul network. Additionally, if the traffic load is high, the operator will mostly benefit from the multiplexing gain at the BBU pool.

The more functionality is moved from the BBU pool to the cell site, the lower the multiplexing gain on the BBU pool. However, when traffic starts to be variable bit rate, a multiplexing gain on fronthaul links can be achieved, lowering the required capacity. Hence, for low traffic load, and even more for bursty traffic, the BBU pool should only have higher layer processing and then the requirements on the fronthaul link can be relaxed. Deployments with hybrid functional splits have been evaluated in order to give indications on how much of the traffic can be carried on specific interface. The analysis is done such that the operator would be able to balance the cost for BBU pool, fronthaul link capacity and resource utilization.

For UE-Cell split we observed a high impact on multiplexing gain value depending on multiplexing gain calculation method, i.e. using different throughput averaging windows. We verified the application level delays for different aggregated link bit rates and thereby we concluded what is the practical value of multiplexing gain that can be achieved. Studying the CDFs of throughput we proposed rules of thumb for network/BBU dimensioning. The more video traffic is present in the network, the delays are more sensitive to the aggregated link bandwidth, and thereby to the multiplexing gain.

\section{REFERENCES}

[1] J. Wu, Z. Zhang, Y. Hong, and Y. Wen, "Cloud radio access network (CRAN): A primer," Network, IEEE, vol. 29, pp. 35-41, Jan 2015.

[2] A. Checko, H. L. Christiansen, Y. Yan, L. Scolari, G. Kardaras, M. S Berger, and L. Dittmann, "Cloud RAN for Mobile Networks - a Technology Overview," IEEE Communications Surveys \& Tutorials, IEEE, vol. 17, pp. 405-426, Firstquarter 2015.

[3] D. Wubben, P. Rost, J. Bartelt, M. Lalam, V. Savin, M. Gorgoglione, A. Dekorsy, and G. Fettweis, "Benefits and impact of cloud computing 
on $5 \mathrm{~g}$ signal processing: Flexible centralization through cloud-ran," Signal Processing Magazine, IEEE, vol. 31, pp. 35-44, Nov 2014.

[4] "Small Cell Virtualization Functional Splits and Use Cases ," tech. rep., Small Cell Forum, June 2015.

[5] P. Rost, C. Bernardos, A. Domenico, M. Girolamo, M. Lalam, A. Maeder, D. Sabella, and D. Wubben, "Cloud technologies for flexible 5g radio access networks," Communications Magazine, IEEE, vol. 52, pp. 68-76, May 2014.

[6] "Next Generation Fronthaul Interface," tech. rep., China Mobile Research Institute, June 2015.

[7] "C-RAN The Road Towards Green RAN," tech. rep., China Mobile Research Institute, October 2011.

[8] "White Paper of Next Generation Fronthaul Interface," tech. rep., China Mobile Research Institute, Alcatel-Lucent, Nokia Networks, ZTE Corporation, Broadcom Corporation, Intel China Research Center, October 2015.

[9] U. Dotsch, M. Doll, H.-P. Mayer, F. Schaich, J. Segel, and P. Sehier, "Quantitative analysis of split base station processing and determination of advantageous architectures for LTE," vol. 18, pp. 105-128, June 2013.

[10] C. Chen, "The Notion of overbooking and Its Application to IP/MPLS Traffic Engineering," Request for Comments: internet draft < draft-cchente-overbooking-01.txt>, November 2001.

[11] Robert B. Cooper and Daniel P. Heyman, "Teletraffic Theory and Engineering," Encyclopedia of Telecommunications, vol. 16, no. 1, pp. 453483, 1998.

[12] V. B. Iversen, Teletraffic Engineering. Chapter 7: Multi-dimensional loss systems. Technical University of Denmark, 2013.

[13] M. Stasiak, M. Głąbowski, A. Wiśniewski, and P. Zwierzykowski, Modeling and dimensioning of mobile networks : from GSM to LTE. John Wiley \& Sons Ltd., 2011.

[14] T. Werthmann, H. Grob-Lipski, and M. Proebster, "Multiplexing gains achieved in pools of baseband computation units in $4 \mathrm{~g}$ cellular networks," in Personal Indoor and Mobile Radio Communications (PIMRC), 2013 IEEE 24th International Symposium on, pp. 3328-3333, Sept 2013.

[15] S. Bhaumik, S. P. Chandrabose, M. K. Jataprolu, A. Muralidhar, V. Srinivasan, G. Kumar, P. Polakos, and T. Woo, "CloudIQ: A framework for processing base stations in a data center," Proceedings of the Annual International Conference on Mobile Computing and Networking, MOBICOM, pp. 125-136, 2012

[16] S. Namba, T. Matsunaka, T. Warabino, S. Kaneko, and Y. Kishi, "ColonyRAN architecture for future cellular network," in Future Network Mobile Summit (FutureNetw), 2012, pp. 1-8, July 2012.

[17] M. Madhavan, P. Gupta, and M. Chetlur, "Quantifying multiplexing gains in a wireless network cloud," in Communications (ICC), 2012 IEEE International Conference on, pp. 3212-3216, 2012.

[18] J. Liu, S. Zhou, J. Gong, Z. Niu, and S. Xu, "On the statistical multiplexing gain of virtual base station pools," in Global Communications Conference (GLOBECOM), 2014 IEEE, pp. 2283-2288, Dec 2014.

[19] A. Checko, H. Holm, and H. Christiansen, "Optimizing small cell deployment by the use of C-RANs," in European Wireless 2014 (EW 2014).

[20] A. Avramova, H. Christiansen, and V. Iversen, "Cell deployment optimization for cloud radio access networks using teletraffic theory," in The Eleventh Advanced International Conference on Telecommunications, AICT 2015.

[21] V. B. Iversen, "The Exact Evaluation of Multi-Service Loss Systems with Access Control," Teleteknik, English ed., vol. 31, pp. 56-61, Firstquarter 1987.

[22] X. Cheng, "Understanding the characteristics of internet short video sharing: Youtube as a case study," in Procs of the 7th ACM SIGCOMM Conference on Internet Measurement, San Diego (CA, USA), 15, p. 28, 2007.

[23] J. J. Lee and M. Gupta, "A new traffic model for current user web browsing behavior," tech. rep., Intel, 2007.

[24] “Average Web Page Breaks 1600K." [cited: June 2015].

[25] A. Checko, L. Ellegaard, and M. Berger, "Capacity planning for carrier ethernet lte backhaul networks," in Wireless Communications and Networking Conference (WCNC), 2012 IEEE, pp. 2741-2745, April 2012.

[26] Metro Ethernet Forum, "EVC Ethernet Services Definitions Phase 3, Tech. Spec. MEF 6.2," July 2014

[27] “Mobility Report," tech. rep., Ericsson, June 2015.

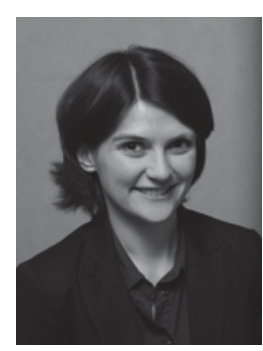

Aleksandra Checko holds a M.Sc. degrees in telecommunication from Technical University of Denmark (DTU) and Technical University of Lodz, Poland (PŁ) (2011). She is now pursuing industrial Ph.D. studies with DTU Fotonik (in the Networks Technology and Service Platforms Group) and MTI Radiocomp. She participated in a Danish national project SAIRS and a European project HARP. Her interests include mobile networks, especially their architecture, protocols, synchronization and capacity planning methods.

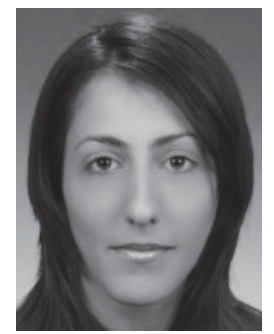

Andrijana P. Avramova has received her M.Sc. degree in telecommunication from the Technical University of Denmark (DTU) in 2008. She is now pursuing Ph.D. studies with DTU Fotonik (in the Networks Technology and Service Platforms Group). She has participated in a Danish national project SAIRS Her research interests are mainly related to heterogeneous mobile networks, especially resource management and cross tier optimizations.

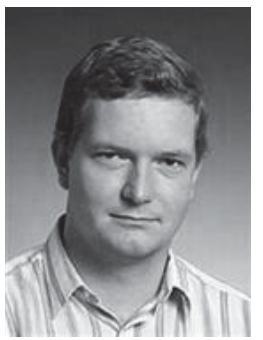

Michael S. Berger received the M.Sc. E.E. and Ph.D. from the Technical University of Denmark in 1998 and 2004. He is currently Associate Professor at the university within the area of switching and network node design. He has been involved in the IST project ESTA (Ethernet at 10 Gigabit and Above), IST project MUPBED. Previously, he was leading a project on next generation IP and Carrier Ethernet networks partly funded by the Danish National Advanced Technology Foundation. He is currently Dissemination Manager in EU project MODUS and board member of several national research projects.

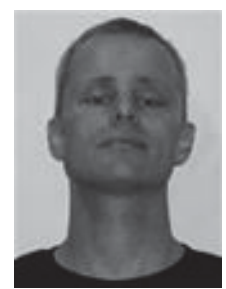

Henrik L. Christiansen is an Associate Professor in mobile communication at the Technical University of Denmark, where he also received his M.Sc.E.E. and $\mathrm{Ph} . \mathrm{D}$., specializing in telecommunications. $\mathrm{He}$ also has several years of experience from the telecom industry. His main areas of research are mobile network architectures, mobile fronthaul and backhaul networks. 